\title{
Quantum breathers in lithium tantalate ferroelectrics
}

\author{
Arindam Biswas $\cdot$ Sutapa Adhikar $\cdot$ Kamal Choudhary $\cdot$ \\ Reshmi Basu • A. K. Bandyopadhyay · A. K. Bhattacharjee • \\ D. Mandal
}

Received: 3 April 2012/ Accepted: 30 April 2012/Published online: 6 June 2012

(C) The Author(s) 2012. This article is published with open access at Springerlink.com

\begin{abstract}
Lithium tantalate is technologically one of the most important ferroelectric materials with a low poling field that has several applications in the field of photonics and memory switching devices. In a Hamiltonian system, such as dipolar system, the polarization behavior of such ferroelectrics can be well-modeled by Klein-Gordon (K-G) equation. Due to strong localization coupled with discreteness in a nonlinear K-G lattice, there is a formation of breathers and multi-breathers that manifest in the localization peaks across the domains in polarization-spacetime plot. Due to the presence of nonlinearity and also impurities (as antisite tantalum defects) in the structure, dissipative effects are observed and hence dissipative breathers are studied here. To probe the quantum states related to discrete breathers, the same K-G lattice is quantized to give rise to quantum breathers $(\mathrm{QBs})$ that are explained by a periodic boundary condition. The gap between the localized and delocalized phonon-band is a function of impurity content that is again related to the effect of pinning of domains due to antisite tantalum defects in the system, i.e., a point of easier switching within
\end{abstract}

A. Biswas - S. Adhikar · K. Choudhary - R. Basu ·

A. K. Bandyopadhyay ( $\square)$

Dumkal Institute of Engineering and Technology,

W. B. University of Technology, Dumkal 742406, India

e-mail: asisbanerjee1000@gmail.com

K. Choudhary

Department of Materials Science and Engineering,

University of Florida, Gainesville, FL 32611, USA

A. K. Bhattacharjee - D. Mandal

Department of Electronics and Communication Engineering,

National Institute of Technology, Durgapur 713209, India the limited amount of data on poling field, which is related to Landau coefficient (read, nonlinearity). Secondly, in a non-periodic boundary condition, the temporal evolution of quanta shows interesting behavior in terms of 'critical' time of redistribution of quanta that is proportional to QB's lifetime in femtosecond having a possibility for $\mathrm{THz}$ applications. Hence, the importance of both the methods for characterizing quantum breathers is shown in these perspectives.

Keywords Ferroelectrics · Quantum breathers · Quantum localization $\cdot$ Temporal evolution of quanta

\section{Introduction}

In the field of applied physics, one of the most investigated materials is ferroelectric, which has important applications as memory switching (Fu and Cohen 2000; Lines and Glass 1977; Kim et al. 2002; Bandyopadhyay and Ray 2004) and in nonlinear optical communications (Gahagan et al. 1999), non-volatile memory devices (Dawber et al. 2005; Catalan et al. 2007), and many others (Kim et al. 2001; Bandyopadhyay et al. 2010). Lithium tantalate with a low poling field appears to be a promising candidate as a key photonic material for a variety of devices: such as optical parametric oscillators, nonlinear frequency converters, electro-optics and second-order nonlinear optical material, holography, etc. Many of such applications include important nanodevices (Bandyopadhyay et al. 2010; Waser 2005; Giri et al. 2011a).

Ferroelectricity is an electrical phenomenon whereby certain materials exhibit a spontaneous dipole moment. The direction of this moment can be switched between the equivalent states by the application of an external electric 
field (Fu and Cohen 2000; Lines and Glass 1977; Kim et al. 2002; Bandyopadhyay and Ray 2004). It arises in certain crystal systems that undergo second-order structural changes below the Curie temperature, which results in the development of spontaneous polarization. This can be explained by Landau-Ginzburg free energy functional (Kim et al. 2002; Bandyopadhyay and Ray 2004; Bandyopadhyay et al. 2010). The ferroelectric behavior is commonly explained by the rotation of domains and domain walls that are present in the crystal with uniform polarization (Fu and Cohen 2000; Lines and Glass 1977; Kim et al. 2002; Bandyopadhyay and Ray 2004). This behavior is nonlinear in terms of hysteresis of polarization $(P)$ and electric field $(E)$ vectors.

Ferroelectricity arises due to the collections of domains, where the ferroelectric domains, as with ferromagnetic domains, are created and oriented by a need to minimize the fields as well as the free energy of the crystal. The bulk properties and domain structure of these materials have been extensively studied (Kim et al. 2002; Bandyopadhyay and Ray 2004; Bandyopadhyay et al. 2010; Srinivas and Vu-Quoc 1995; Bandyopadhyay et al. 2006a, b, 2008, Scrymgeour et al. 2005). However, recently they have gained renewed interest for potential applications in nanoscience and the design of nanodevices, where the focus is on properties exhibited at small length scales (Bandyopadhyay et al. 2010; Giri et al. 2011a). The new applications are based on both classic ferroelectric properties and features that are particular to nano-structured arrays and include applications to (Waser 2005): integrated or surface-mounted capacitors, electromechanical sensors, actuators, transducers, infrared sensors, tunable thermistors, and nonlinear dielectric materials. Due to these current interests, we look for details of the dynamical properties of domain arrays, which may become significant features at some of the length scales of interest, and this aspect is definitely very important.

Several studies have been made by Meyer and Vanderbilt (2002) on the ab initio calculation of energy of the domain wall having a narrow width of the order of lattice spacing as well as on defect pinning of domain wall in lead titanate (He and Vanderbilt 2003). By powerful NMR experiments on intrinsic defects of ferroelectrics, Yatsenko et al. (1998) did an impressive study on domain dynamics of lithium and antisite niobium defects structure. Lee et al. (2009) used density functional theory (DFT) approach and molecular dynamics simulations to show that the $180^{\circ}$ domain walls have mixed character in lead titanate and lithium niobate that can be dramatically enhanced in nanoscale thin film heterostructures where the internal wall structure can form polarization vortices. They also showed (Xu et al. 2009) the behavior of Er defects in lithium niobate by energetics and stability considerations by DFT combined with thermodynamic calculations, which yields exclusively the role of defects on the charge balance and not direct relevance to the ferroelectric domains or domain walls. Quantum chemical calculations were also undertaken by Stashans et al. (Stashans et al. 2006) on the oxygen vacancy defects in lead titanate crystals. It should be mentioned that the relaxor ferroelectrics and intrinsic inhomogeneity were also studied by Bussman-Holder et al. (2000) for dielectrically soft matrix.

Phenomenological level of description has been used in many previous theoretical and experimental investigations of ferroelectric domain walls, particularly by Dawber et al. (2005) and Catalan et al. (2007). First principle calculations have also been performed on ferroelectrics by Klotins et al. (Klotins 2010) in terms of nanopolar regions. Some of these investigations have been made to get an overview of the domains and domain walls in ferroelectrics in terms of 'smaller length scales' in which the excitations can exist. As the domains are small and discrete in the nano-range, some discussion is necessary on localization in ferroelectrics to understand the meaning of classical and quantum breathers.

Localization in a system is attributed either to its disorder or to its nonlinearity (Fleischer et al. 2003). The first one, i.e., Anderson localization has been implemented in details in many types of device applications. As the nonlinearity arises in ferroelectrics in terms of $P-E$ hysteresis due to the rotational movement of the discrete domains and domain walls, they could also give rise to the localization. Hence, the above explanations are given to relate the localized waves of discrete breathers and domain walls in ferroelectric materials. Moreover, having explained the inter-relation between domains and discrete breathers, it should be pointed out that as the rotation of domain and domain wall is important for switching and again, as switching is influenced by pinning of defects on the domain walls in ferroelectrics (Kim et al. 2002; Scrymgeour et al. 2005), it was considered useful to deal with discrete breathers through our discrete Hamiltonian (Bandyopadhyay et al. 2006a, 2010). This Hamiltonian gives rise to nonlinear $\mathrm{K}-\mathrm{G}$ equation with a damping term to take care of dissipation, as it is expected that the antisite tantalum defects also tend to promote the nature of dissipation in the ferroelectric system.

The above description also shows the importance of domain wall in ferroelectrics in describing a soliton solution, i.e., nonlinear localized traveling waves that are robust and propagate without change in shape, giving the polarization profile and the distribution of the elastic strain across the domain wall (Dauxois and Peyrard 2006). On the other hand, discrete breathers (DBs) are discrete solutions, periodic in time and localized in space and whose frequencies extend outside the phonon spectrum (Flach and 
Gorbach 2008; Sato et al. 2006). This is described by our discrete Hamiltonian (Bandyopadhyay et al. 2006a, 2010), as detailed later.

As also indicated in Dauxois and Peyrard (2006), the ferro-para phase change occurs through a global and coordinate displacement of the ions. Hence, the presence of solitons is due to the Landau double well potential in which the tantalum ions are sitting with their coupling that is strong enough to lead to cooperative effects. However, these motions become spatially localized due to nonlinearity and discreteness along with the pinning effect of the ferroelectric domains and domain walls, which are typically in the "nano-range". Therefore, the essence of nonlinearity and discreteness paves the way for the nanoferroelectric devices (Waser 2005; Giri et al. 2011a). Then, there are other aspects, such as quantum computation and targeted energy transfer (TET) by using a new concept through DBs (Maniadis et al. 2004). Hence, DBs assume more significance obviously in the context of 'localization'. Due to inhomogeneous nature of ferroelectrics, a pinning effect also contributes to the localization phenomenon. The combined effects seem to be the basis of localization in ferroelectrics that gives rise to the formation of DBs which has not been described in our previous work on discreteness (Bandyopadhyay et al. 2006a, 2010). After discussing about classical breathers, next let us look for quantum breathers.

In this new approach, for the characterization of DBs or classical breathers (Giri et al. 2011b), the bulk system was the right tool, but when we are dealing with smaller systems, we have to use quantum physics, which brings us to the quantum breathers (QBs) (Scott et al. 1994; Pinto et al. 2009). Once generated, QBs modify system properties such as lattice thermodynamics and introduce the possibility of non-dispersive energy transport, as generally described for DBs (Tsironis 2003). These are observed in many systems, viz. ladder array of Josephson junction for superconductors, BEC in optical lattices/nonlinear photonic lattices, interacting optical waveguides, cantilever vibrations in micromechanical arrays, DNA, split-ring resonator (SRR) based metamaterials in antenna arrays, two-magnon bound states in antiferromagnets, two-phonon bound states (TPBS), i.e., quantum breathers, in ferroelectrics (see Biswas et al. 2011a).

A brief account is given here on phonon bound state or breather state. Despite our work on discrete breathers (Bandyopadhyay et al. 2010; Giri et al. 2011b), so far the pinning has been explained classically, thereby prompting us to think about quantum explanations that have been briefly explored for ferroelectrics, such as lithium niobate with the impurity data (Biswas et al. 2011a). Although impurity data for lithium tantalate (as antisite tantalum defects) are not available, we could work through Landau coefficient or nonlinearity route to explore if there is also pinning in such systems (see later). For quantum breathers, it is important to consider detailed information on phonons and their bound-state concept, which is sensitive to the degree of nonlinearity. In the eigenspectrum or more traditionally $E_{k}$ versus $k$ plot, a quantum breather band separates itself from the delocalized phonon-band. Or in other words, it is the hopping tendency of the phonons that describe the quantum breathers. So, let us consider that the phonons in one sublattice may hop from one domain to another adjacent domain. This hopping might have some consequences with the change of nonlinearity or poling field for 'switching' that is again related to the impurity in the lattice, thereby the 'hopping strength' can be directly related to this phenomenon. It is determined by finding the phonon-band energy gap (i.e., the energy gap between the delocalized and localized phonons) in the usual eigenspectrum (Flach and Gorbach 2008; Nguenang et al. 2007).

In quantum mechanics, single phonons are considered delocalized. If two phonons are bound, then it can be in a localized state that is a necessity for the formation of quantum breathers. For these two types of phonons, as the energy is different, the two-phonon localized band has to separate out of the single-phonon continuum. As the phonons are quantized vibrations, the breathers thus formed through spatial localization and localized excitations or oscillations in the nano-scale are called "quantum breathers" (QBs). Therefore, the two-phonon bound states (TPBS) are considered as signature of QBs. The evidence of TPBS was shown in an interesting work by Cohen and Ruvalds (Cohen and Ruvalds 1969). A femtosecond timedomain analog of light-scattering spectroscopy called impulsive stimulated Raman scattering (ISRS) is a very useful technique that has been extensively used by Brennan et al. (Brennan and Nelson 1997) to deal with the anharmonic vibrations in both lithium niobate and lithium tantalate crystals.

Thus, the goal of this paper is to explore whether the switching is easier at particular point of 'nonlinearity' and also 'coupling' within the domains by quantum calculations hitherto not done on lithium tantalate. Therefore, the hopping strength of phonon and thus the phonon energy gap have been derived from the quantized model of the ferroelectric system. The calculations of various TPBS parameters are made against Landau coefficients (read, nonlinearity) to highlight the quantum origin of pinning in lithium tantalate with important consequences for various nano-ferroelectric devices. So far, we have described a situation for TPBS route by a periodic boundary condition. Next, let us also discuss about QB in a non-periodic boundary condition.

QBs are also characterized by various methods (Pinto and Ricardo 2008; Schulman et al. 2006). Here our main focus will be on the temporal evolution of the number of 
quanta, as it is convenient to characterize quantum breathers by this method. It has to be noted that in an anharmonic model such as K-G lattice, the potential energy levels are non-equidistant. This makes us properly harness the physical as well as computational behavior (Flach and Gorbach 2008; Nguenang et al. 2007). Thus, this aspect could have important implications from the point of view of application. QBs have been studied for dimer and trimer cases, and that also by (mainly) periodic boundary condition approach. However, a real material consists of many subunits, i.e., thousands of domains make ferroelectrics, each acting as sites and phonons act here as bosons or quanta. Again, how the increase of number of sites and bosons affects a system can also be regarded as an interesting topic. Hence, it drives us to a study that considers more number of sites and quanta. This is also the main aim of this paper.

Quantum localization behavior in K-G lattice has been studied by many researchers in terms of four atom lattice with periodic function, notably by Proville (2005), dimer case for targeted energy transfer by Maniadis et al. (2004), delocalization and spreading behavior of wave-packets by Skokos et al. (2009). Here we present a generalized method for any number of sites and quanta without periodic boundary condition to show the QB states. In K-G lattice, it is important to calculate the 'critical time' of redistribution of quanta under various physical conditions. It is the "time" when the temporal evolution of the number of quanta first meets or tends to meet.

For strengthening our focus on temporal evolution of quanta, it has to be noted that the application of $\mathrm{QB}$ in ferroelectrics consists of many different fields of technology, namely 'phase-coherent optical pulse synthesis' (Shelton et al. 2001), 'parametric light generation' (Ebrahimzadeh 2003), 'ultrafast spectroscopy' (Rousse et al. 2001), etc. On the latter application, extensive investigations by Brennan and Nelson (1997) on both lithium tantalate and lithium niobate are quite important. The critical point at which the temporal evolution of quanta meets or tends to meet may be directly related to ferroelectric switching phenomenon, which if tailored well could lead to any of the above applications. Hence, viewing ferroelectricity in terms of phonons and its study via temporal evolution under various controlling parameters assume significance.

The paper is organized as follows: in "Theoretical development", we first present some details of spectral collocation method to develop space-time evolution of polarization plots for overall view of classical breathers in "K-G equation and classical breathers", then we present the general mathematical model for TPBS parameters and finally after second quantization on K-G lattice is done with Bosonic operators along with our method of computation in "Quantum breathers". In "Results and discussion", the results and discussion are also presented in three parts for the above three cases. In "Conclusion", the conclusions are given.

\section{Theoretical development}

$\mathrm{K}-\mathrm{G}$ equation and classical breathers

We consider an idealized model of a one-dimensional array of $N$ identical ferroelectric domains layered along the $x$ axis (Bandyopadhyay et al. 2006a, 2010). In the ground state the array of polarized domains is antiferroelectrically ordered. Here, the domains are in the $x$-direction, of height $d \ll N L_{1}$ in the $z$-direction, and translationally invariant in the $y$-direction. The domain length $\left(L_{1}\right)$ is typically of the order of a few hundred nanometers. Between the neighboring domains, there are domain walls of length $W_{L} \ll L_{1}$ and $d$ of the order of a few nanometers. The total length of the array along the $x$-direction is $L_{2}=N L_{1}$. For simplicity the polarization of each domain is oriented along $z$-direction; the domain arrangement is shown in Bandyopadhyay et al. (2010). In a previous treatment, a time-dependent formulation for the dynamics of the domain array was obtained as a generalization of the Landau-Ginzburg free energy form involving polarization $(P)$ and electric field (E) vectors (Bandyopadhyay et al. 2006a). The nearest neighbor domains [i.e., the polarization in the $i$ th domain $\left(P_{i}\right)$ with that in the $(i-1)$ th domain $\left.\left(P_{i-1}\right)\right]$ were taken to interact by a harmonic potential with a phenomenological spring constant $k$ so that the resulting Hamiltonian for the polarization is given by (Bandyopadhyay et al. 2006a, 2010):

$$
\begin{aligned}
H= & \sum_{i=1}^{N}\left(\frac{1}{2 m_{d}}\right) p_{i}^{2}+\sum_{i=1}^{N} \frac{k}{4}\left(P_{i}-P_{i-1}\right)^{2} \\
& +\sum_{i=1}^{N}\left(\left(-\frac{\alpha_{1}}{2} P_{i}^{2}+\frac{\alpha_{2}}{4} P_{i}^{4}\right)-E P_{i}\right)
\end{aligned}
$$

The momentum $\left(p_{i}\right)$ can be defined in terms of order parameter $\left(P_{i}\right)$ as:

$p_{i}=\frac{\partial}{\partial t}\left(\frac{m_{d}}{Q_{d}} P_{i}\right)=\left(\frac{m_{d}}{Q_{d}}\right) \dot{P}_{i}$

where $m_{d}$ and $Q_{d}$ are two inertial constants. For developing the K-G equation the index $(i)$ is dropped. Equation (1) gives a good general treatment of the mode dynamics in the array, particularly for modes, which are strongly localized over a small number of the domains in the array. For extended modes and modes which are localized, and slowly range over a large number of consecutive domains, Eq. (1) can be approximated by a continuum treatment by Taylor 
expansion. In this limit, expressed in dimensionless units, Eq. (1) yields a nonlinear K-G equation with a damping term (Bandyopadhyay et al. 2006a, 2010) as:

$$
\frac{\partial^{2} P}{\partial t^{2}}+\bar{\gamma}-\frac{\partial P}{\partial t}-\bar{k}\left(\frac{\partial^{2} P}{\partial x^{2}}\right)-\left(\bar{\alpha}_{1} P-\bar{\alpha}_{2} P^{3}\right)-E_{0} \cos (\omega t)=0
$$

for the dynamics of polarization $P(x, t)$. Here, Eq. (2) with an ac driver $\left(E=E_{0} \cos (w t)\right)$ contains all the nondimensional terms as: $P^{\prime}=P / P_{\mathrm{s}}$, where $P_{\mathrm{s}}$ is the saturation polarization in $\mathrm{C} / \mathrm{m}^{2}$ [typical value for lithium tantalate ferroelectrics as $0.55 \mathrm{C} / \mathrm{m}^{2}$ (Kim et al. 2002)], $E^{\prime}=E / E_{\mathrm{c}}$, where $E_{\mathrm{c}}$ is the coercive field (when $P^{\prime}=0$ ) in $\mathrm{kV} / \mathrm{cm}$ in the usual nonlinear hysteresis curve of $P$ versus $E$ with a typical value for the same material as $17 \mathrm{kV} / \mathrm{cm}$ (Kim et al. 2002), $t^{\prime}=t / t_{\mathrm{c}}$, where $t_{\mathrm{c}}$ is considered as the critical time scale for polarization to reach a saturation value, i.e., at or near the domain walls that are of importance to our study with a typical value of $10 \mathrm{~ns}$ for a switching time of (say) $200 \mathrm{~ns}$ for a damping value $\bar{\gamma}=$ 0.50 (Bandyopadhyay et al. 2006b) that are based on the above data, and $x^{\prime}=x / W_{L}$, where $W_{L}=$ domain wall width of the order of a few nm. Equation (1) is obtained after dropping the prime notation, and by taking $\alpha_{1}=\alpha_{2} / P_{\mathrm{s}}^{2}$ and $\bar{\alpha}_{1}=\bar{\alpha}_{2}=\bar{\alpha}=\left(\alpha_{1} P_{\mathrm{s}}\right) / E_{\mathrm{c}} \quad$ (Kim et al. 2002; Bandyopadhyay et al. 2010). Here, the interaction and damping terms are defined as:

$\bar{k}=\frac{k P_{\mathrm{s}}}{2 E_{\mathrm{c}}}$

and

$\bar{\gamma}=\frac{\gamma P_{\mathrm{s}}}{t_{\mathrm{c}} E_{\mathrm{c}}}$

where $\gamma$ is a decay constant relating the loss of polarization due to damping (Giri et al. 2011a) during its motion in the system of domains, which is important for the motion of localized traveling waves (i.e., solitons). Although discrete breathers rides on the background of phonon, the phonons are themselves dispersive in nature and the nonlinear systems are also dispersive that is coupled with discreteness (Rasmussen et al. 1998). Moreover, the ferroelectric switching involves the domain rotation that gives rise to dispersion. This is the reason of including the 'damping term' in our spectral collocation method of describing 3D pictures of polarization profile (see "Quantum breathers").

Among all the known methods of numerical simulation, we use the most versatile method of spectral collocation to analyze classical breathers in our system of ferroelectrics by using K-G equation. This method is not only the latest numerical technique with ease of implementation, but also gives rise to a minimum of errors in the analysis. Spectral methods are a class of spatial discretizations for differential equations. The key components for their formulation are trial functions and the test functions (Canuto 2006). Now, the nonlinear discrete $\mathrm{K}-\mathrm{G}$ equation in the $i$ th domain can be given by:

$$
\begin{aligned}
& \frac{\partial^{2} P_{i}}{\partial t^{2}}+\bar{\gamma} \frac{\partial P_{i}}{\partial t}-\bar{k}\left(P_{i-1}-2 P_{i}+P_{i+1}\right)-\bar{\alpha}\left(P_{i}-P_{i}^{3}\right) \\
& \quad-E_{0} \cos (\omega t)=0 .
\end{aligned}
$$

Here, $P_{i}$ is the polarization of the $i$ th domain and $\bar{k}$ denotes the interaction or coupling parameter incorporating the lattice distance term. To prepare the equation for numerical solution we introduce the auxiliary variable: $Q_{i}=\dot{P}_{i}=\frac{\partial P_{i}}{\partial t}$. This reduces the second-order Eq. (5) to the first order system:

$\dot{P}=Q$

$\dot{Q}=\bar{k} D P-\bar{\gamma} Q+\bar{\alpha}\left(P-P^{3}\right)+E_{0} \cos (\omega t)$.

The notation $i$ is dropped for subsequent computation work, as shown above. An appropriate banded matrix $D$ has to be selected (Wiedeman and Reddy 2000) and then we solve our system of the first order differential equations that can be written as:

$$
\begin{aligned}
\left(\begin{array}{c}
\dot{P} \\
\dot{Q}
\end{array}\right)= & \left(\begin{array}{cc}
I & 0 \\
-\bar{\gamma} & \bar{k} D
\end{array}\right)\left(\begin{array}{c}
Q \\
P
\end{array}\right)+\bar{\alpha}\left(\begin{array}{c}
0 \\
\left(P-P^{3}\right)
\end{array}\right) \\
& +\left(\begin{array}{c}
0 \\
U
\end{array}\right) E_{0} \cos (\omega t) .
\end{aligned}
$$

Here, $U$ is a column vector whose elements are unity. We have used well-known fourth order Runge-Kutta method for the system (8).

Next, we consider the DBs subjected to losses due to damping and the external driving force $E_{0} \cos (\omega t)$, i.e., for dissipative DBs. The term $\bar{k}$ represents the interaction term and the Landau term $\bar{\alpha}$ (anharmonic parameter) is a characteristic feature of our ferroelectric system. As emphasized in Kim et al. (2002), the Landau term is very important in deciding the formulation of discrete breathers (see later in Figs. 1 and 2). Next, let us deal with QBs in terms of TPBS parameters in a periodic boundary condition.

\section{Quantum breathers}

\section{Periodic boundary condition approach}

Equation (1) gives a general treatment of the mode dynamics in the array, particularly for modes, which are strongly localized over a small number of domains in the array. For extended modes and modes which are localized, and slowly range over a large number of consecutive domains, our discrete Hamiltonian (Eq. 1) can be split as: 


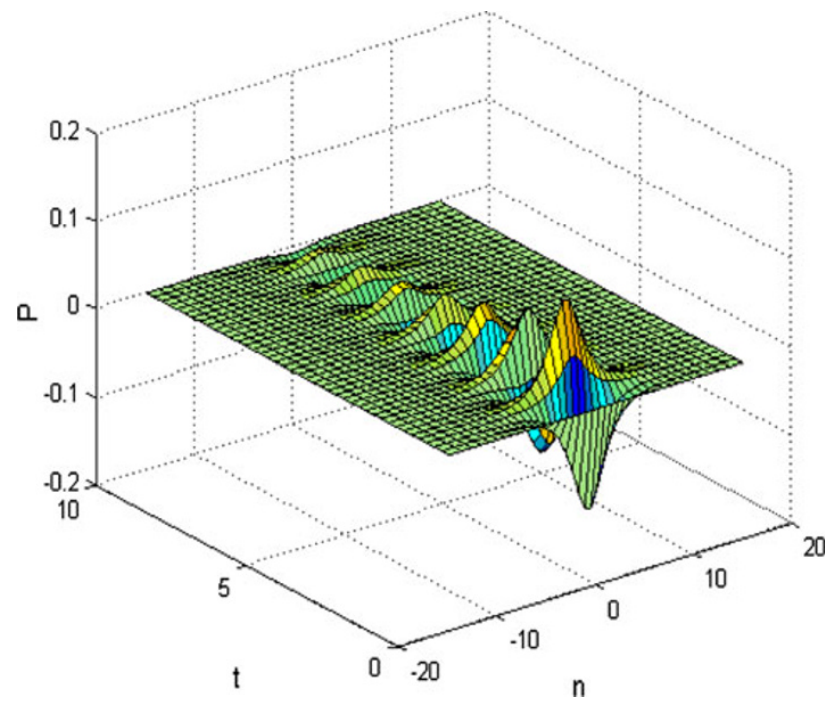

Fig. 1 Dissipative breathers in lithium tantalate with a smaller value of coupling for a poling field of $E_{\mathrm{c}}=17 \mathrm{kV} / \mathrm{cm}$, nonlinearity $\bar{\alpha}=420.76$, coupling $\bar{k}=0.5$, damping $\bar{\gamma}=0.5$, and the driving field $E=0.10$ for symmetric DBs. Without changing the values of poling field and nonlinearity, the effect of increasing damping is observed at higher value when symmetric breathers almost disappears. Here, all the data are dimensionless

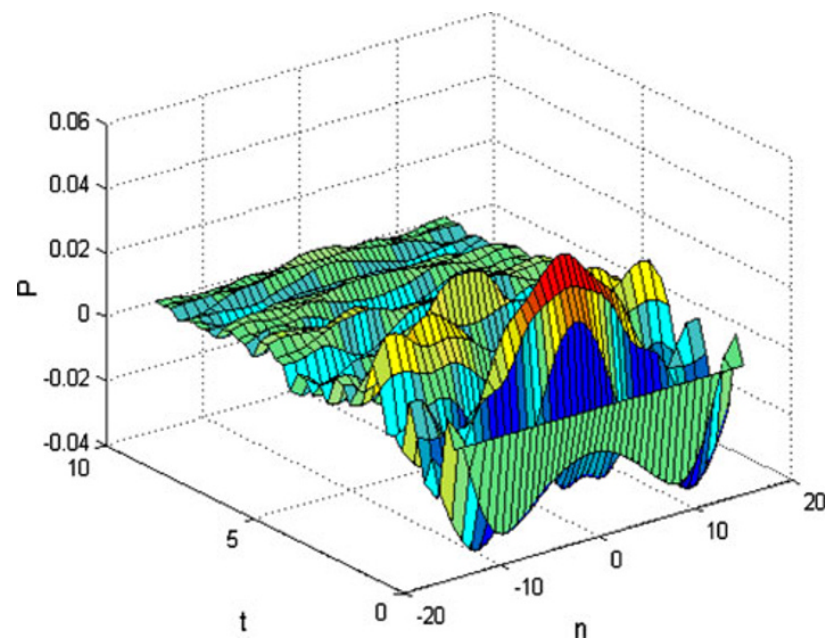

Fig. 2 Formation of tri-breathers in lithium tantalate with a substantial increase of coupling constant for $E_{\mathrm{c}}=17 \mathrm{kV} / \mathrm{cm}$, $\bar{\alpha}=420.76, \bar{k}=60, \bar{\gamma}=0.5, E=0.10$ to show the effect of coupling in forming multi-breathers. Here, all the data are dimensionless

$\tilde{H}=H_{0}+H_{1}$

where, the two components of the Hamiltonian are written as:

$H_{0}=\sum_{i} \frac{p_{i}^{2}}{2}-\frac{\left(\alpha_{1}+\lambda\right) P_{i}^{2}}{2}+\frac{\alpha_{2} P_{i}^{4}}{4}-E P_{i}$

$H_{1}=-\frac{\lambda}{2} \sum_{i} P_{i} P_{i-1}$.
Here, $p_{i}$ is the momentum of the system, as defined in Eq. (1a). Hence, for $n$-particles or $n$-levels in the anharmonic potential well, a general basis may be written as:

$$
\begin{aligned}
|\vec{n}\rangle & =\psi_{n_{1}}\left(x_{1}\right) \psi_{n_{2}}\left(x_{2}\right) \ldots \psi_{n_{f}}\left(x_{f}\right) \text { and }\langle\vec{m}|H| \vec{n}\rangle \\
& =\left\langle\vec{m}\left|H_{0}\right| \vec{n}\right\rangle+\left\langle\vec{m}\left|H_{1}\right| \vec{n}\right\rangle .
\end{aligned}
$$

For the present numerical analysis, the Fourier grid Hamiltonian method (Nguenang et al. 2007) with 1,000 grids and 0.006 spacing to calculate various eigenvalues and eigenvectors at the grid points. For the present calculations, we restrict ourselves to two-phonon states, since at the working temperature the number of phonon is very small. In order to reduce the computer memory requirement, we take the advantage of translational invariance by periodic Bloch wave formulation, as detailed in Biswas et al. (2011a) and Nguenang et al. (2007).

The energy gap between the single-phonon continuum and a bound state is given by:

$E_{\mathrm{g}}=E_{2}-E_{0}-2\left(E_{1}-E_{0}\right)$

where $E_{0}, E_{1}$ and $E_{2}$ are three eigenvalues at different points of wave vector $(k)$ that are calculated from our computation to generate $E(k)$ versus $k$ curve, which gives the signature of quantum breathers $(\mathrm{QB})$ in terms of twophonon bound state. The width of the single phonon in the eigenspectrum is given by the magnitude of $4 \sigma$ and the width of the single delocalized phonon-band, where $\sigma$ is expressed as:

$\sigma=-\frac{\lambda}{2} D_{01}^{2}$

where $\lambda$ is an interaction term (i.e., equivalent to $k$ ). $D_{01}$ represents the coefficient for zero to single-phonon generation. The variation of the single-phonon spectrum width $\left(W_{\mathrm{ph}}\right)$ represents (through $D_{01}=D_{10}$ ) the creation of a new phonon or annihilation of an existing phonon. Again, the hopping coefficient for a single phonon to become a two-phonon bound state is given by:

$\mu=-\frac{\lambda}{2} D_{01} D_{12}=-\frac{\lambda}{2} D_{10} D_{21}$.

All the above calculations were done for 51 sites or domains and $\lambda=10$. More data points could be used in our present simulation, but here we are primarily focused to study nonlinearity/impurity induced critical behavior of discrete breather motion and its quantum origin. To treat the problem analytically we take the help of second quantization method. By quantizing the Hamiltonian in Eq. (1) in a number conserving quantized form with $N \rightarrow \infty$ (Flach and Gorbach 2008) with $\lambda_{1}=\frac{\lambda}{(\lambda-\alpha)}, \eta=$ $\frac{2 \alpha}{(\lambda-\alpha)}$ and $E=0$ in Eq. (1) leads to the equation for twophonon amplitudes as: 
$\hat{H}=\sum_{n} a_{n}^{+} a_{n}+\frac{3}{8} \eta a_{n}^{+2} a_{n}^{2}+\frac{\lambda_{1}}{2}\left\{a_{n}^{+}\left(a_{n+1}+a_{n-1}\right)+\right.$ h.c. $\}$

where $a_{n}^{+}$and $a_{n}$ are the creation and annihilation operators, and h.c. is the Hermitian conjugate; the terms $\eta$ and $\lambda_{1}$ are already defined above.

Hence, for a fixed total quasi-momentum $K$, the critical $\alpha$-value or Landau parameter may be calculated as:

$\alpha_{\text {TPBS }}=\lambda\left[\frac{\cos \frac{K R}{2}}{E_{V}-1}-1\right]$.

Here $R$ is a lattice parameter and $E_{v}$ is the eigenvalue representing the bound state. Hence, for a lithium tantalate type ferroelectrics, after $\alpha_{\text {TPBS }}$, a branch is separated from the continuum due to corresponding value of impurity content (read, nonlinearity) and has got a critical point for pinning transition. Now, for bound state $m=n$ and the bound-state energy $\left(E_{\mathrm{BS}}\right)$ can be derived as:

$E_{\mathrm{BS}}=1+\frac{3 \eta}{8}-4 \lambda_{1} \cos \frac{K R}{2}$.

Substituting the values of $\eta$ and $\lambda_{1}$ and taking the eigenvalue equal to the energy of the bound state, and also taking the cosine term as equal to 1 , the critical $\alpha$ value or Landau parameter may be related as:

$E_{\mathrm{BS}}=\frac{\alpha}{\alpha-\lambda}$.

The results on the calculation of various TPBS parameters for lithium tantalate are shown in Table 1 and their variation with nonlinearity (i.e., $\alpha$ value) is shown in "Quantum breathers". Next, let us deal with the non-periodic boundary condition approach for QBs.

\section{Non-periodic boundary condition}

The general Hamiltonian for the Klein-Gordon equation for order parameter $\left(y_{n}\right)$ at $n$th site is written as:

$H=\sum_{n} \frac{p_{n}^{2}}{2 m}+\frac{A}{2} y_{n}^{2}+\frac{B}{4} y_{n}^{4}+k\left(y_{n}-y_{n-1}\right)^{2}$.

Table 1 The values of various TPBS parameters in lithium tantalate

\begin{tabular}{lrrr}
\hline $\begin{array}{l}\alpha \text { (n.d.) } \\
\text { (poling field in kV/cm) }\end{array}$ & $\left|E_{\mathrm{g}}\right|$ & $|\sigma|$ & $\mu$ \\
\hline $4,426.68(1.60)$ & 92.45 & 4.90 & 2.475 \\
$513.66(13.90)$ & 30.06 & 4.70 & 0.63 \\
$420.76(17.00)$ & 26.95 & 4.61 & 26.92 \\
$37.09(210.00)$ & 3.44 & 2.38 & 1.48 \\
\hline
\end{tabular}

Poling field in $\mathrm{kV} / \mathrm{cm}$ is shown beside the $\alpha$ value, $k=10$ (coupling), $4 \sigma=W_{\text {ph }}=$ single-phonon hopping strength, $\mu=$ one to two phonon hopping strength. The data in last 3 columns are in electron volts n.d. $=$ non-dimensional
The first term is momentum at $n$th site $\left(p_{n}\right)$, the second and third terms are nonlinear potential formulation and the last term contains an interaction constant $(k)$. From the above Eq. (17), after deducing the classical equation of motion and rescaling of time, we get:

$\tilde{H}=\sum_{n} \frac{1}{2} p_{n}^{2}+\frac{1}{2} y_{n}^{2}+\eta y_{n}^{4}+\lambda\left(y_{n}-y_{n-1}\right)^{2}$

where $\eta=\frac{B}{4 A} \lambda=\frac{k}{2 A}, A$ and $B$ are two constants. Now, let us use creation and annihilation Bosonic operators at the $n$th site and the above Hamiltonian (Eq. 18) is quantized. In an important work done by Proville (2005) the nonnumber conserving methods for four sites and an arbitrary number of particles are shown. However, the method presented above gives a generalized way to solve the system for arbitrary number of particles on arbitrary number of sites. So, our method is clearly distinguished from the other investigations.

After second quantization, a general 'basis' is then created. For the characterization of quantum discrete breathers, we need to make the Hamiltonian time-dependent. Let us take the help of temporal evolution of number of bosons at each site of the system:

$\left\langle n_{i}\right\rangle(t)=\left\langle\Psi_{t}\left|\hat{n}_{i}\right| \Psi_{t}\right\rangle$

We take $i$ th eigenstate of the Hamiltonian, and then we make it time-dependent as follows:

$\left|\Psi_{i}(t)\right\rangle=\sum_{i} b_{i} \exp \left(-i E_{i} t / \hbar\right)\left|\psi_{i}\right\rangle$,

where $\psi_{i}$ and $E_{i}$ is the $i$ th eigenvector and eigenvalue, respectively, and $t$ is time. The Planck's constant $(h)$ is taken as unity and $b_{i}=\left\langle\psi_{i} \mid \psi(0)\right\rangle$ for each site $i$ and for a given range of $t$, where $\psi(0)$ stands for the initial state.

It is pertinent to mention that in contrast with the Discrete Non-Linear Schrodinger (DNLS) equation, where complete energy transfer takes place (Maniadis et al. 2004), in case of nonlinear K-G lattice, complete energy transfer does not take place between the anharmonic oscillators and there is a critical time of redistribution for the quanta (Flach and Gorbach 2008). This is an important point to be noted. With the above methodology, we can now proceed to deal with the applications of non-periodic boundary condition approach in lithium tantalate.

\section{Results and discussion}

In a Hamiltonian system, such as dipolar system, the space-time perspective of polarization can be modeled by Klein-Gordon equation. By spectral method, it is possible to get an overview of the system. In our previous work 
(Bandyopadhyay et al. 2006a, 2010) on discrete Hamiltonian exploring the possibility of formation of discrete breathers, the overall physical overview was not shown and then in Giri et al. (2011b), such diagrams were shown for only lithium niobate. Later, in a short communication, 3D pictures for lithium tantalate were shown for a poling field value of $13.9 \mathrm{kV} / \mathrm{cm}$ (Biswas et al. 2011b). Here, we are showing similar 3D pictures, but at a different poling field value of $17 \mathrm{kV} / \mathrm{cm}$ with more explanations on pinning. All the units in Figs. 1 and 2 are dimensionless. It was not known from quantum point of view whether lithium tantalate could also show the possibility of pinning. Hence, we first investigate the issue here as classical aspect and in the later section the detailed studies encompassing QBs in terms of TPBS parameters. When Klein-Gordon lattice is quantized, we obtain a separation of two-phonon bound states that are localized which are signatures of quantum breather states. These are shown in Figs 3, 4, 5, 6, where all the units are dimensionless. From our computation, three parameters are mainly calculated, i.e,. (1) energy gap between the localized and delocalized single-phonon continuum, (2) width of the single-phonon band and also (3) hopping coefficient, as described in "Periodic boundary condition approach". All these data are shown in Figs. 7, 8, 9, where all the units are in eV. Moreover, for characterizing QBs in terms of temporal evolution of quanta, it is also necessary to make a generalized Hamiltonian timedependent, as described in "Non-periodic boundary condition". This also shows the femtosecond response of such QBs with the possibility of application in the $\mathrm{THz}$ range.

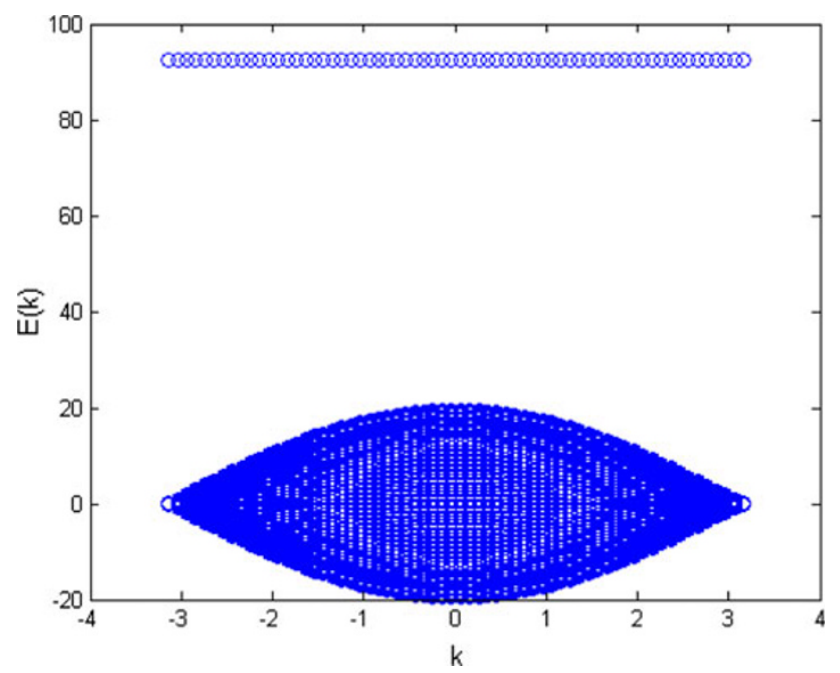

Fig. 3 Eigenspectrum for a coupling value of $\bar{k}=10$, for a low poling field $E_{\mathrm{c}}=1.6 \mathrm{kV} / \mathrm{cm}$ and $\bar{\alpha}=4,427$. The continuum represents single-phonon continua and the quantum breather band or twophonon bound state is in the upper branch of the spectrum. Here, all the data are dimensionless

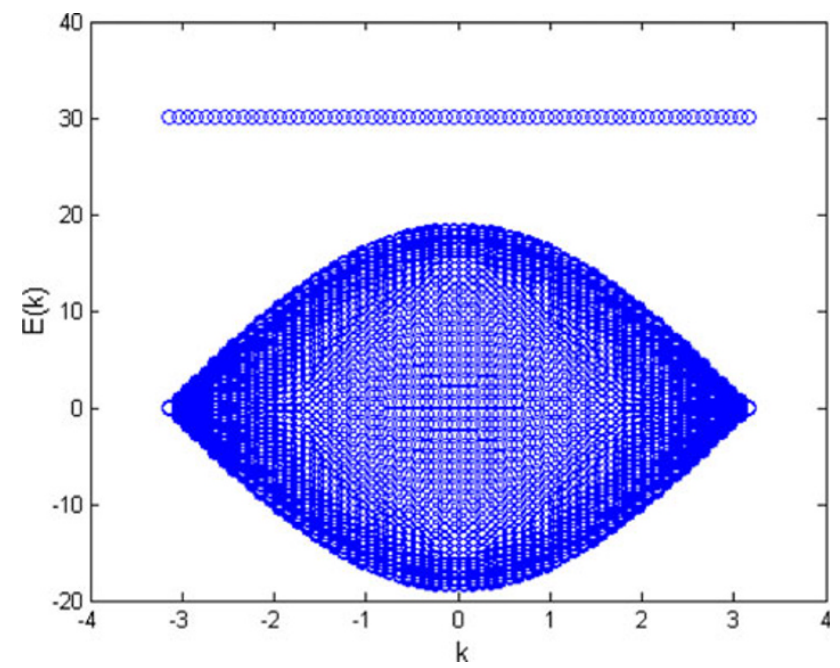

Fig. 4 Eigenspectrum for a coupling value of $\bar{k}=10$, for a moderate poling field $E_{\mathrm{c}}=13.9 \mathrm{kV} / \mathrm{cm}$ and $\bar{\alpha}=514$. The same single-phonon continua and the quantum breather band in the upper branch of the spectrum are clearly observed with the effect of a decrease of nonlinearity in the single-phonon width. Here, all the data are dimensionless

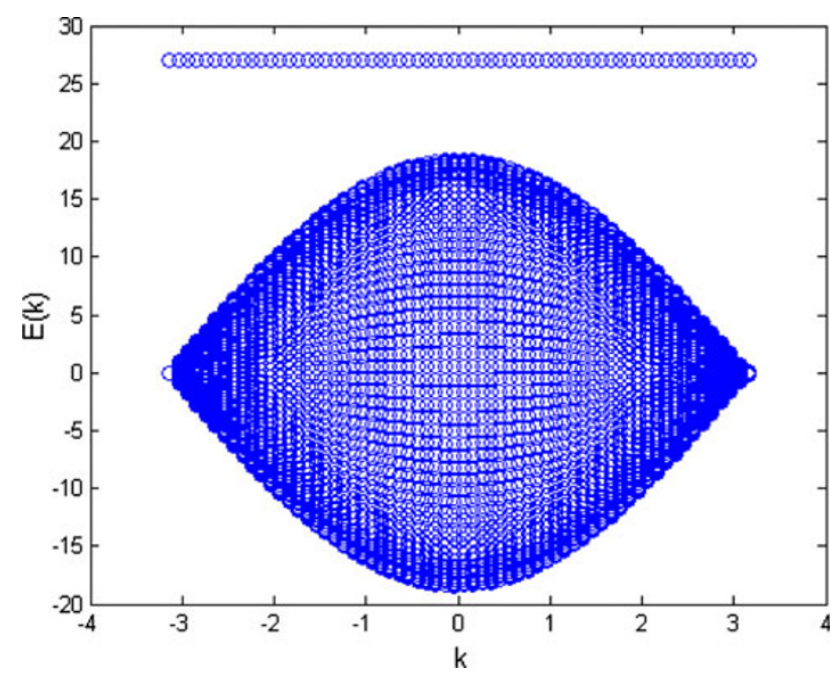

Fig. 5 Eigenspectrum for a coupling value of $\bar{k}=10$, for a moderate poling field $E_{\mathrm{c}}=17 \mathrm{kV} / \mathrm{cm}$ and $\bar{\alpha}=421$. The similar spectra are observed with the effect of a decrease of nonlinearity in the singlephonon width. Here, all the data are dimensionless

\section{Discrete breathers}

Lithium tantalate is a very important class of ferroelectric materials that have been extensively studied due to its usefulness in many nano-structured device applications (Bandyopadhyay et al. 2010; Waser 2005; Giri et al. 2011a). This ferroelectric material has much lower coercive field, say, $17 \mathrm{kV} / \mathrm{cm}$. This is considered more useful in the device applications for easier switching, as the device thickness can still be made higher than that for other 


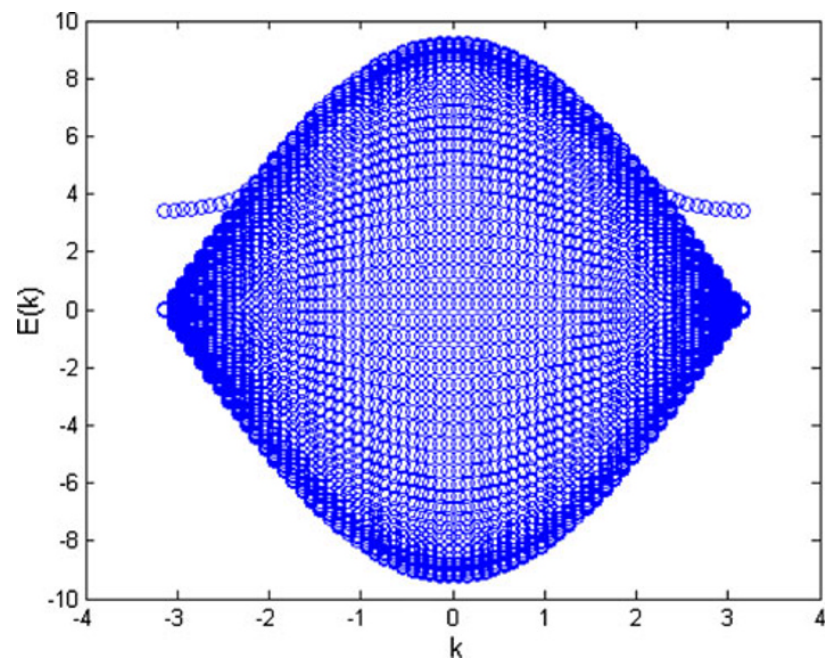

Fig. 6 Eigenspectrum for a coupling value of $\bar{k}=10$, for the highest poling field $E_{\mathrm{c}}=210 \mathrm{kV} / \mathrm{cm}$ and lowest nonlinearity $\bar{\alpha}=37$. The similar spectra are observed with the effect of a further decrease of nonlinearity in the single-phonon width and in the appearance of a pseudo gap. Here, all the data are dimensionless

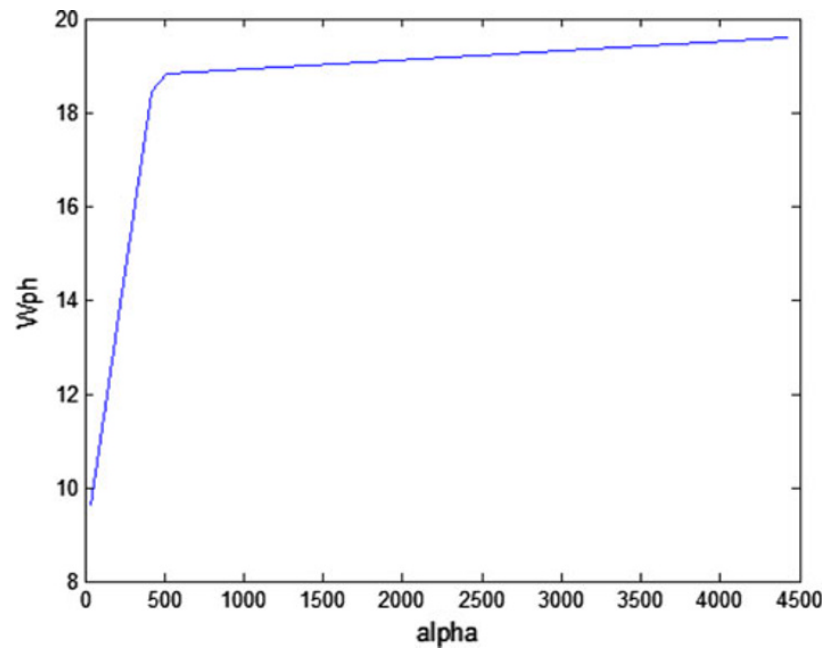

Fig. 7 Variation of the width of the single-phonon continua or the hopping energy $\left(W_{\mathrm{ph}}\right)$ in $\mathrm{eV}$ that is calculated from Eq. (11) is shown against nonlinearity (dimensionless) in lithium tantalate for a constant coupling of 10

crystals. An extensive amount of work has been done by Gopalan and co-workers on lithium tantalate and its switching behavior (Kim et al. 2001, 2002; Yang et al. 1999; Gopalan 2007). Typical 3D polarization diagrams for lithium tantalate are shown in Figs. 1 and 2, respectively, that are considered as manifestations of discrete breathers in our system due to localization, as our analysis is based on discrete domains.

For a case with a zero field and no damping, i.e., Hamiltonian breathers, symmetric breathers are normally observed in 3D figures (not shown). It should be mentioned

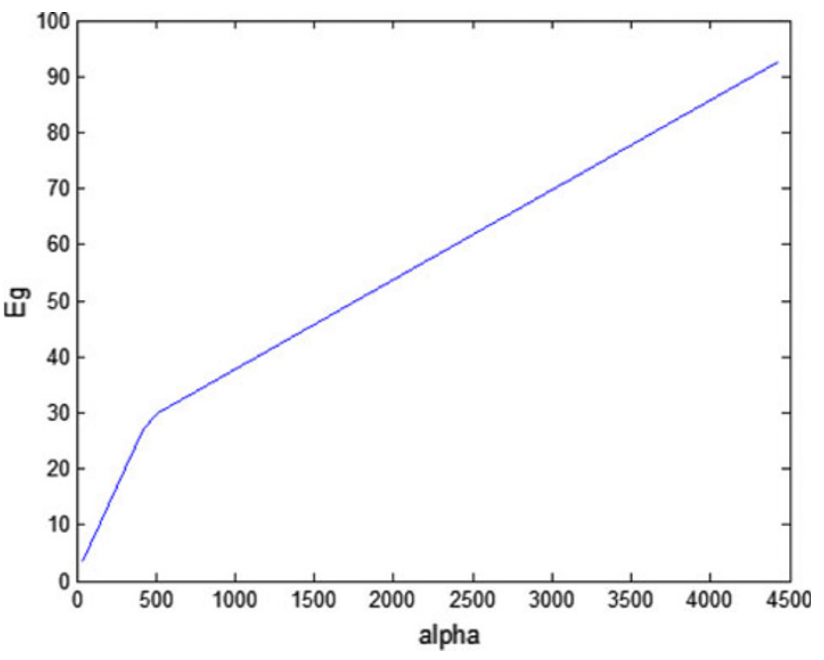

Fig. 8 Variation of the energy gap $\left(E_{\mathrm{g}}\right)$ in $\mathrm{eV}$ that is calculated from Eq. (10) is shown against nonlinearity (dimensionless) in lithium tantalate for a constant coupling of 10

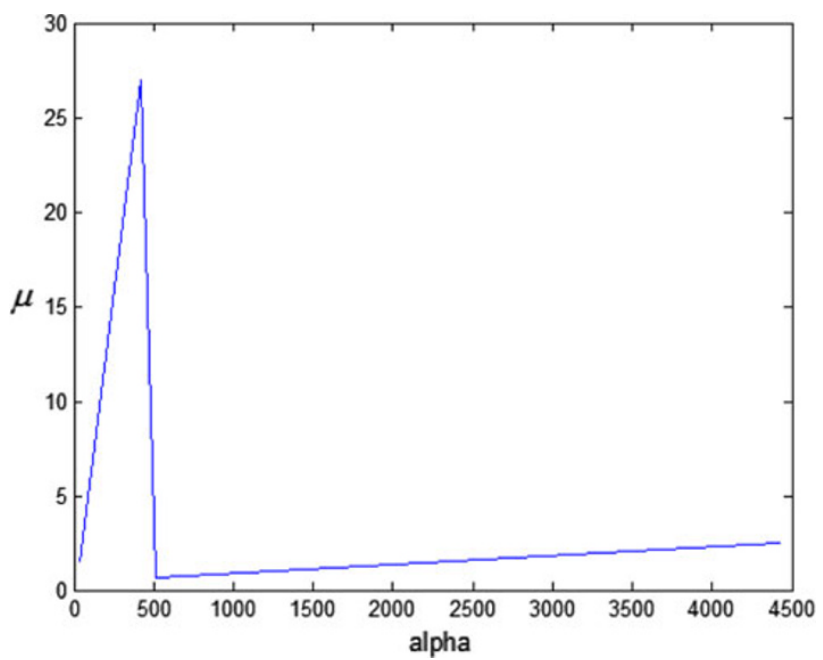

Fig. 9 Variation of the hopping coefficient $(\mu)$ in $\mathrm{eV}$ that is calculated from Eq. (12) is shown against nonlinearity (dimensionless) in lithium tantalate for a constant coupling of 10. It shows a sharp transition for $\bar{\alpha}=421\left(E_{\mathrm{c}}=17 \mathrm{kV} / \mathrm{cm}\right)$

that in the simulation of 2D figures of polarization $(P)$ with site index $(n)$, i.e., distance, the peaks have been found to be symmetric Gaussian bands and even in 3D pictures the same type of symmetric bands are observed that are termed symmetric breathers. However, for a sample with poling field of $17 \mathrm{kV} / \mathrm{cm}$ with a finite value of field $(E=0.01)$ and a moderate damping (0.50) with low level of interaction constant $(0.50)$, it is seen from Fig. 1 that the dissipation starts to visibly show up and the symmetric dissipative DBs are still observed. If the damping value is further increased to a high value of 0.9 , the intensity almost decays to zero which is expected from dissipative 
breathers. With an increasing value of interaction, i.e., at a very high value of 60 , it is seen from Fig. 2 that the "tribreathers" are formed, as also observed in the case of lithium niobate (Giri et al. 2011b). It should be mentioned that the 3D diagrams (Figs. 1,2) are very similar with those shown in Biswas et al. (2011b) with lower poling field value, as the difference between the nonlinearity parameters (513 and 421, respectively), is not as significant as in this analysis.

Such high value of interaction (i.e., up to 50) was also taken to see the behavior of 'mode amplitudes' in our previous study of intrinsic localized modes or DBs (Bandyopadhyay et al. 2010). In this case, the importance of the coupling parameter is also observed in creating multibreathers in lithium tantalate system. However, in our numerical simulation, bi-breathers have already been observed at a lower value of coupling and there is no formation of multi-breathers up to a coupling value of 6 . As coupling increases further, multi-breathers start forming. There are important observations made on the appearance of multisolitons by controlling various parameters by a number of authors with numerical solutions for optical communication devices (Ablowitz and Biondini 1998; Gabitov et al. 2007).

\section{Quantum breathers}

\section{TPBS parameters}

Next, let us present the data for two-phonon bound state (TPBS) or quantum breather (QB) state in lithium tantalate system. It is pertinent to mention that in our recent work on another important nonlinear optical material, such as splitring-resonator (SRR) based metamaterial, both K-G equation and nonlinear Schrodinger equation (NLSE) show dark and bright solitons, and also dark and bright breathers. However, K-G equation in addition shows breather pulses, whereas NLSE does not show such pulses (Giri et al. 2011c). This work could also be relevant for an important nonlinear optical material, such as lithium tantalate in the future directions of study, where K-G equation seems to show interesting behavior.

The eigenspectra showing TPBS in lithium tantalate are shown in Figs. 3, 4, 5, 6 for a poling field value of $1.60,13.90,17$ and $210 \mathrm{kV} / \mathrm{cm}$, respectively. The coupling value is taken as constant at 10 for all the spectra. The Landau parameter $(\alpha)$ is inversely proportional to the impurity content or poling field. These data along with those of TPBS parameters are shown in Table 1. In the absence of the impurity data, we would operate through Landau coefficient (read, nonlinearity) which should also give a correct picture of QBs in terms of various TPBS parameters, as nonlinearity and discreteness give rise to quantum localization or rather to the formation of QBs.

It is clearly seen from Figs. 3, 4, 5, 6 that as the nonlinearity decrease, i.e., the poling field increases, the shape of the single-phonon continuum also changes in terms of its width that could be considered as significant, as it shows a functional dependence on nonlinearity. This width is measured by $W_{\mathrm{ph}}$ as shown in Equ. (11) which shows a slight decrease towards lower $\alpha$ values $(\alpha=514$ to 421 , i.e., average value $=467$ ) and then it drops quite drastically towards lower $\alpha$ values, i.e., the highest poling field of $210 \mathrm{kV} / \mathrm{cm}$, as shown in Fig. 7. This is in contrast to that in lithium niobate system, where it sharply drops towards $\alpha=471$ and then it sharply increases towards lower $\alpha$ values (Biswas et al. 2011a). This may be due to the different levels of the degree of polarization in the respective systems (Yang et al. 1999). It also indicates that in lithium niobate, as the nonlinearity decreases from 1,767 towards 471, the difference in values of $W_{\mathrm{ph}}$ is about 19.2 in absolute term, whereas that for lithium tantalate system, it is only about 1 for nonlinearity values from 4,427 to 467 (average). For this phenomenal change despite having a higher level of variation in nonlinearity, the drop in values of the width in the single-phonon continuum is extremely small (see Table 1) implying that the effect of nonlinearity is relatively smaller. It is quite significant for lithium tantalate, which is gaining popularity as an important candidate as device material that is also supplemented by its lower value of poling field for switching. This might indicate that irrespective of the impurity content in lithium tantalate as antisite tantalum defects, there does not seem to be an appreciable effect of pinning in the system. It should be emphasized here that 'pinning' is a macroscopic phenomena involving the charge defects in the system (Yang et al. 1999; Gopalan 2007), whereas TPBS parameters are microscopic in nature that are obtained after detailed quantum calculations. However, defect structure of ferroelectrics also needs to be understood. Another powerful theory, such as density function theory (DFT) as done by some authors (Lee et al. 2009) and also first principle calculations (Klotins 2010) could be useful, but it is beyond the scope of this paper.

Next, let us look into the other TPBS parameters, namely the energy gap $\left(E_{\mathrm{g}}\right)$ that should indicate a different trend to that shown in Fig. 7 above. The relation between the energy gap that is calculated from Eq. (10) and nonlinearity is shown in Fig. 8. It is seen that the energy gap decreases drastically towards an average value of nonlinearity of 467 and thereafter it drops relatively less towards lower $\alpha$ values, i.e., toward the highest poling field of $210 \mathrm{kV} / \mathrm{cm}$, as expected. This might signify that as the nonlinearity decreases in this system, the formation of QB becomes more and more difficult and up to a certain value, 
the switching will also be much easier in the system and thereafter it becomes relatively more difficult, as revealed by quantum calculations of TPBS parameters. It also indicates a possibility of pinning transition, even if the effect may be relatively smaller. It is seen from Fig. 9 that the hopping coefficient $(\mu)$ that is calculated from Equ. (12) against nonlinearity, there is a sharp transition at a poling field of $17 \mathrm{kV} / \mathrm{cm}$ with a nonlinearity value of 421 . For QB under periodic boundary condition, this behavior of the microlevel properties, such as TPBS parameters, can be considered quite significant for future applications. This supports our quantum calculations in that the tendency of phonon hopping to the second excited state may be considered to be showing a direction towards interpretation of some physical behavior of lithium tantalate for some applications in devices.

\section{Temporal evolution of number of quanta}

For a non-periodic boundary condition, a typical simulation for a smaller value of coupling of 0.9 and nonlinearity of 420.76 is shown in Fig. 10. This simulation was done with 4 particles on 3 sites with initial condition as: $|\Psi(0)\rangle=|3,1,0\rangle$. The curve with higher intensity that represents the first site is clearly visible, but that with the lower intensity represents the 'merged' spectra for the other two sites. In our computer simulation work, we have observed that the spectra sometimes get merged in this manner possibly due to the faster process of redistribution of quanta in lithium tantalate system that was not observed in lithium niobate.

For these spectra and others at different quanta, the initial localization is mainly at the first site and then there is a fast redistribution of quanta between other two sites until they become equal or almost equal. Here, the critical time for redistribution $\left(t_{\mathrm{re}}\right)$ that is proportional to QB's lifetime is around $1,359 \mathrm{fs}$. As the number of quanta increases from 6 to 12 , the QB's lifetime decreases significantly to: 421 ,

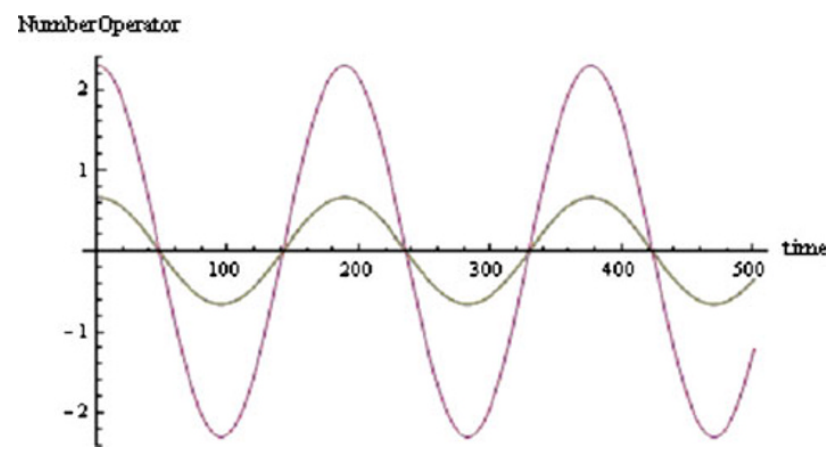

Fig. 10 Temporal evolution spectra for lithium tantalate for 4 particles on 3 sites with $|\Psi(0)\rangle=|3,1,0\rangle$, where there is an overlap of the second and third spectra. The $x$-axis has to be multiplied by 3.048 to get fs
207, 127 and 93 fs, respectively. It is noteworthy that for 6 quanta in lithium niobate, the QB's lifetime is higher at $441 \mathrm{fs}$ for the same value of coupling of 0.9 , whereas that for lithium tantalate under the same condition it is $421 \mathrm{fs}$. A lower lifetime of QBs in the latter case might make it suitable candidate for certain devices.

The above behavior is shown in Fig. 11, wherein it is seen that as the number of quanta increases, the 'critical time of redistribution' continuously decreases. This effect is more pronounced in case of lithium tantalate than that observed in lithium niobate. The data for QB's lifetime in fs for $\alpha=420.76$ for four different values of coupling are shown in Table 2. It is seen that at each value of quanta, the QB's lifetime decreases as the coupling increases, even between 0.1 and 0.9 . This change is particularly noticeable for lower quanta at 4 and 6, respectively. From 8 quanta onwards, the effect seems to be relatively less. Hence, it is observed that the QB's lifetime is relatively more sensitive in case of lithium tantalate than that in lithium niobate.

It is pertinent to mention that for a given macromolecule, Tretiak et al. (2002) observed that the lifetime of discrete breathers increases as the crystals become more and more defective. As the defect or disorder could create localization, it is suitable for the formation of QB giving us insight on quantum localization (Scott et al. 1994; Pinto et al. 2009). The above discussion includes the effect of impurities, as it depends on nonlinearity parameter which in turn is embodied in our Hamiltonian. Therefore, this is discussed in "TPBS parameters" in terms of TPBS parameters and in "Temporal evolution of number of quanta" in terms of temporal evolution. Finally it should be mentioned that the application of $\mathrm{QB}$, particularly in terms

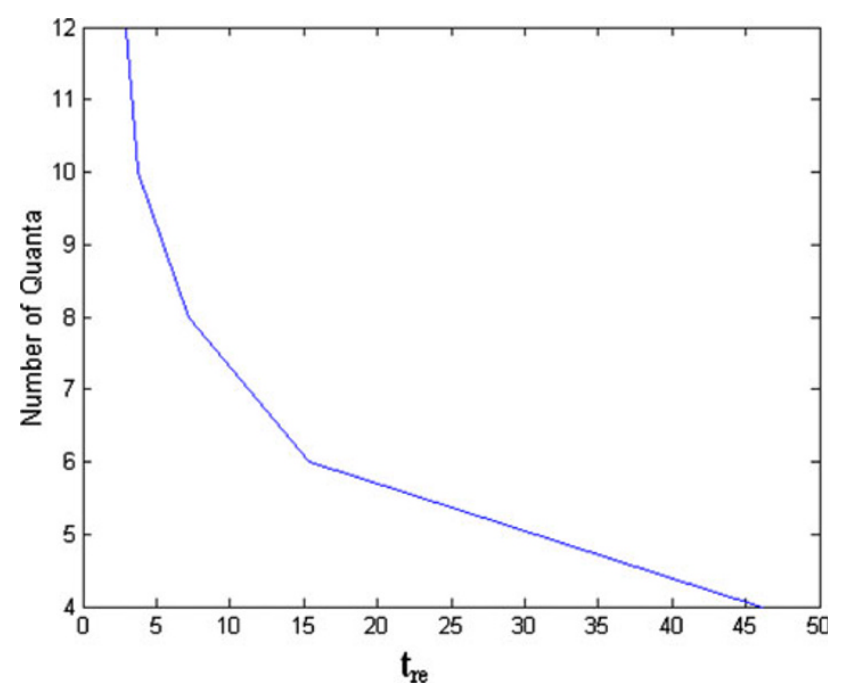

Fig. 11 Number of quanta against the time of redistribution data showing a continuous decrease of this critical time that is proportional to QB's lifetime with the increase of number of quanta. The $x$-axis has to be multiplied by 3.048 to get fs 
Table 2 QB's lifetime in fs for four different values of coupling at $\alpha=420.76$

\begin{tabular}{lcccc}
\hline No. of quanta & Coupling $=0.10$ & Coupling $=0.90$ & Coupling $=10$ & Coupling $=60$ \\
\hline 4 & 4,196 & 1,359 & 396 & 147 \\
6 & 1,262 & 421 & 120 & 48 \\
8 & 620 & 207 & 60 & 24 \\
10 & 380 & 1,027 & 39 & 16 \\
12 & 278 & 93 & 28 & 10 \\
\hline
\end{tabular}

of temporal evolution of quanta, could assume significance if novel technologies and methods are to be adopted, as discussed earlier (Brennan and Nelson 1997; Shelton et al. 2001; Ebrahimzadeh 2003; Rousse et al. 2001).

\section{Conclusion}

The present study of the evolution of polarization with both time and space gives rise to the nonlinear Klein-Gordon equation that is based on a discrete Hamiltonian. By spatial discretization technique via spectral collocation method, the Klein-Gordon equation gives rise to classical breathers as shown for an overall 3D view that are sensitive towards higher damping, or rather the oscillations of the symmetric breathers seem to be quite stable up to a relatively moderate value of damping $(\sim 0.50)$. For quantum breathers in lithium tantalate, in a periodic boundary condition approach, the second quantization gives rise to some interesting values of TPBS parameters against nonlinearity within the system. This shows that after a value of nonlinearity (421), i.e., equivalent to a poling field of $17 \mathrm{kV} /$ $\mathrm{cm}$, the formation of quantum breathers starts becoming relatively difficult thereby increasing single-phonon hopping energy that is observed up to this poling field and thereafter it increases monotonically toward higher values, whereas the energy gap after showing a small increase up to this poling field increases rapidly towards higher values; this was in contrast to that shown in lithium niobate system. The hopping coefficient shows a sharp transition at this point indicating some sort of pinning in this system as well. Without the impurity data in this system, it is difficult to specify the role of antisite tantalum defects. For a nonperiodic boundary condition approach, the temporal evolution spectra show that with increasing number of quanta, there is a decrease of time of redistribution that is proportional to the QB's lifetime in fs. It also shows a strong dependence on the coupling. This piece of information is considered useful for a future study in this new field of investigation of quantum breathers in ferroelectrics and other applications of QBs in important nonlinear optical materials.

Acknowledgments The authors would like to thank Dr S. Flach of Max Planck Institute (MPI) at Dresden (Germany) for his help during computational work. The authors would like to thank Professor Keith Nelson of Department of Chemistry at MIT at Boston (USA) for sharing some ISRS data on lithium tantalates.

Open Access This article is distributed under the terms of the Creative Commons Attribution License which permits any use, distribution, and reproduction in any medium, provided the original author(s) and the source are credited.

\section{References}

Ablowitz MJ, Biondini G (1998) Multiscale pulse dynamics in communication systems with strong dispersion management. Opt Lett 23:1668-1670

Bandyopadhyay AK, Ray PC (2004) Perturbation analysis and memory in ferroelectric materials. J Appl Phys 95:226-230

Bandyopadhyay AK, Ray PC, Gopalan V (2006a) An approach to Klein-Gordon equation for dynamic study in ferroelectric materials. J Phys Condens Matter 18:4093-4099

Bandyopadhyay AK, Ray PC, Gopalan V (2006b) Dynamical systems analysis for polarization in ferroelectrics. J Appl Phys 100:114106-114109

Bandyopadhyay AK, Ray PC, Gopalan V (2008) Solitons and critical break-up fields in lithium niobate type uniaxial ferroelectrics. Eur Phys J B 65:525-531

Bandyopadhyay AK, Ray PC, Vu-Quoc L, McGurn AR (2010) Multiple-time-scale analysis of nonlinear modes in ferroelectric $\mathrm{LiNbO}_{3}$. Phys Rev B 81:064104-064114

Biswas A, Choudhary K, Bandyopadhyay AK, Bhattacharjee AK, Mandal D (2011a) Quantum pinning-transition due to charge defects in ferroelectrics. J Appl Phys 110:024104-024108

Biswas A, Choudhary K, Bandyopadhyay AK, Bhattacharjee AK, Mandal D (2011b) Dissipative breathers modes in nano-ferroelectrics. J Electron Devices 10:423-428

Brennan CJ, Nelson KA (1997) Direct time-resolved measurement of anharmonic lattice vibrations in ferroelectric crystals. J Chem Phys 107:9691-9694

Bussman-Holder A, Bishop AR, Egami T (2000) Relaxor ferroelectrics and intrinsic inhomogeneity. Euro Phys Lett 71:249-255

Canuto C (2006) Spectral methods: fundamentals in single domains. Springer, New York, p 424

Catalan G, Schilling A, Scott JF, Greg JM (2007) Domains in threedimensional ferroelectric nanostructures: theory and experiments. J Phys Condens Matter 19:132201-132207

Cohen MH, Ruvalds J (1969) Two-phonon bound states. Phys Rev Lett 23:1378-1381

Dauxois T, Peyrard M (2006) Physics of solitons. Cambridge University Press, Cambridge, p 211

Dawber M, Rabe KM, Scott JF (2005) Physics of thin-film ferroelectric oxides. Rev Mod Phys 77:1083-1130

Ebrahimzadeh M (2003) Parametric light generation. Phil Trans R Soc A 361:2731-2750 
Flach S, Gorbach AV (2008) Discrete breathers-advances in theory and applications. Phys Rep 467:1-116

Fleischer JW, Segev M, Elfremidis NK, Christodoulides DN (2003) Observation of 2-dimensinal discrete solitons in optically induced nonlinear photonic lattices. Nature 422:147-150

Fu H, Cohen RE (2000) Polarization rotation mechanism for ultrahigh electromechanical response in single-crystal piezoelectrics. Nature 403:281-283

Gabitov I, Indik R, Mollenauer LF, Shkarayev M, Stepanov M, Lushnikov PM (2007) Twin families of bisolitons in dispersionmanaged systems. Opt Lett 32:605-607

Gahagan KT, Gopalan V, Robinson JM, Jia QX, Mitchell TE, Kawas MJ, Schlesinger TE, Stancil DD (1999) Integrated electro-optics lens/scanner in a $\mathrm{LiTaO}_{3}$ single crystal. Appl Opt 38:1186-1190

Giri P, Ghosh S, Choudhary K, Alam Md, Bandyopadhyay AK, Ray PC (2011a) Importance of damping on nanoswitching in $\mathrm{LiNbO}_{3}$ type ferroelectrics. Phys Scr 83:015702-015706

Giri P, Choudhary K, Sengupta A, Bandyopadhyay AK, Ray PC (2011b) Discrete breathers in nonlinear $\mathrm{LiNbO}_{3}$-type ferroelectrics. J Appl Phys 109:054105-054112

Giri P, Choudhary K, Sengupta A, Bandyopadhyay AK, McGurn AR (2011c) Klein-Gordon equation approach to non-linear split-ring resonator based metamaterials: 1D systems. Phys Rev B 84:155429_ 155510

Gopalan V (2007) Defect domain wall interactions in trigonal ferroelectrics. Ann Rev Mater Res 37:449-489

He L, Vanderbilt D (2003) First-principles study of oxygen-vacancy pinning of domain walls in $\mathrm{PbTiO}_{3}$. Phys Rev B 68:134103134109

Kim S, Gopalan V, Kitamura K, Furukawa Y (2001) Domain reversal and nonstoichiometry in lithium tantalate. J Appl Phys 90:2949-2963

Kim S, Gopalan V, Gruverman A (2002) Coercive fields in ferroelectrics: a case study in lithium niobate and lithium tantalate. Appl Phys Lett 80:2740-2742

Klotins E (2010) Intrinsic localized excitations: heuristic explanations for the polar nanoregions. Phys E 42:614-617

Lee D, Behera RK, Wu P, Xu H, Li YL, Sinnott SB, Phillpot SR, Chen LQ, Gopalan V (2009) Mixed Bloch-Néel-Ising character of $180^{\circ}$ ferroelectric domain wall. Phys Rev B 80:060102-060105

Lines ME, Glass AM (1977) Principles and applications of ferroelectrics and related materials. Clarendon, Oxford

Maniadis P, Kopidakis G, Aubry S (2004) Classical and quantum targeted energy transfer between nonlinear oscillators. Phys D 188:153-177

Meyer B, Vanderbilt D (2002) Ab initio study of ferroelectric domain walls in $\mathrm{PbTiO}_{3}$. Phys Rev B 65:104111-104121

Nguenang JP, Pinto RA, Flach S (2007) Quantum q-breathers in a finite Bose-Hubbard chain: the case of interacting bosons. Phys Rev B 75:214303-214308

Pinto RA, Ricardo A (2008) Quantum breathers in capacitively coupled Josephson junctions: correlation, number conserving and entanglement. Phys Rev B 77:024308-024310
Pinto RA, Haque M, Flach S (2009) Edge-localized states in quantum 1-dimensional lattices. Phys Rev A 79:052118-052125

Proville L (2005) Biphonons in the Klein-Gordon lattice. Phys Rev B 71:104306-104311

Rasmussen KO, Christiansen PL, Johansson M, Gaididei YuB, Mingaleev SF (1998) Localized excitations in discrete nonlinear Schrödinger systems: effects of nonlocal dispersive interactions and noise. Phys D 113:134-151

Rousse A, Rischel C, Gauthier J (2001) Femtosecond X-ray crystallography. Rev Mod Phys 73:17-31

Sato M, Hubbard BE, Sievers AJ (2006) Nonlinear energy localization and its manipulation in micromechanical oscillator arrays. Rev Mod Phys 78:137-157

Schulman LS, Tolkunov D, Mihokova E (2006) Structure and time dependence of quantum breathers. Chem Phys 322:55-74

Scott AC, Eilbeck JC, Gilhøj H (1994) Quantum lattice solitons. Phys D 78:194-213

Scrymgeour DA, Gopalan V, Itagi A, Saxena A, Swart PJ (2005) Phenomenological theory of a single domain wall in uniaxial trigonal ferroelectrics: lithium niobate and lithium tantalate. Phys Rev B 71:184110-184122

Shelton RK, Ma LS, Kapteyn HC, Murnane MM, Hall JL, Ye J (2001) Phase-coherent optical pulse synthesis from separate femtosecond lasers. Science 293:1286-1289

Skokos Ch, Krimer DO, Komineas S, Flach S (2009) Delocalization of wave packets by disordered nonlinear chains. Phys Rev E 79:056211-056212

Srinivas V, Vu-Quoc L (1995) Accurate phenomenological models for all four phases of $\mathrm{BaTiO}_{3}$ via semi-infinite optimization. Ferroelectrics 163:29-57

Stashans A, Serrano S, Medina P (2006) A quantum-chemical study of oxygen vacancy defects in lead titanate crystals. Physica B 381:82-89

Tretiak S, Saxena A, Martin RL, Bishop AR (2002) Conformational dynamics of photoexcited conjugated molecules. Phys Rev Lett 89:97402-97405

Tsironis GP (2003) If discrete breathers is the answer, what is the question? Chaos 13:657-666

Waser R (ed) (2005) Nanoelectronics and information technology. Wiley, Weinhiem

Wiedeman JAC, Reddy SC (2000) A MATLAB differentiation matrix suite. ACM Trans Math Softw (TOMS) 26:465-519

Xu H, Lee D, Sinnott SB, Gopalan V, Dierolf V, Phillpot SR (2009) Structure and energetics of Er defects in $\mathrm{LiNbO}_{3}$ from first principles and thermodynamic calculations. Phys Rev B 80:144104-144114

Yang TJ, Gopalan V, Swart PJ, Mohideen U (1999) Direct observation of pining and bowing of a single ferroelectrics domain wall. Phys Rev Lett 82:4106-4109

Yatsenko AV, Ivanova-Maksikova HM, Sergeev NA (1998) NMR study of intrinsic defects in congruent $\mathrm{LiNbO}_{3}$. 2. Overlapping defects. Phys B 254:256-259 